\title{
A reassessment of the risk of multiple sclerosis developing in patients with optic neuritis after extended follow-up
}

\author{
D A FRANCIS, ${ }^{*} \dagger$ D A S COMPSTON, $\ddagger$ J R BATCHELOR, ${ }^{*}$ W I MCDONALD $\dagger$
}

From the Department of Immunology, Royal Postgraduate Medical School, ${ }^{*}$ London, Institute of Neurology, National Hospital for Nervous Diseases, $\dagger$ London, and the Section of Neurology, University of Wales, College of Medicine, $\ddagger$ Heath Park, Cardiff, UK

SUMMARY One hundred and one of 146 patients presenting with isolated idiopathic optic neuritis, previously reviewed in 1978, were reassessed clinically, and retyped for HLA antigens and Factor B alleles, after a mean follow-up of 11.6 years. Fifty eight patients (57\%) had developed multiple sclerosis at the time of reassessment in the present study, of whom $51(88 \%)$ had clinically definite disease. This compared with $40 \%$ of the original group, in 1978 , of whom $62 \%$ then had clinically definite multiple sclerosis. When the life-table method of analysis was used, the probability of developing multiple sclerosis was $75 \%, 15$ years after the initial episode of optic neuritis. The frequencies of HLA-DR2 and the recently defined D-region antigen, DQw1, were significantly increased in patients with isolated optic neuritis and those who subsequently developed multiple sclerosis compared with normal controls, but neither allele appears to influence progression from optic neuritis to multiple sclerosis. Patients with optic neuritis who were HLA-DR3 positive had an increased risk for the development of multiple sclerosis $(R R=2 \cdot 8)$ and this risk was further enhanced when DR3 occurred in combination with DR2 $(R R=6 \cdot 7)$. The overall increased risk of developing multiple sclerosis for patients with this combination was 26 times that for the normal population. When the patients' original tissue-typing was considered BT 101 no longer influenced conversion of optic neuritis to multiple sclerosis. This may partly be explained by improved methods of tissue-typing, since not all BT 101 positive patients were subsequently found to be positive for HLA-DR2 or HLA-DQw1 and vice versa and by extended follow-up as multiple sclerosis conversion in HLA-DR2 negative individuals increased with time. All 101 patients were typed for Factor B alleles. No significant differences in frequencies were found between individuals with isolated optic neuritis or those who progressed to multiple sclerosis compared with the control population. Recurrent episodes of optic neuritis were not associated with an increased risk for the development of multiple sclerosis in this study.

There is general agreement that a proportion of patients presenting with unilateral optic neuritis eventually develop multiple sclerosis; ${ }^{1}$ what remains uncertain is the percentage of individuals that follow this course and when the conversion is likely to occur. Most European studies report that the incidence of

Address for reprint requests: Professor W I McDonald, Institute of Neurology, Queen Square, London WCI 3BG, UK.

Received 11 November 1986

Accepted 5 December 1986 multiple sclerosis after optic neuritis is $50 \%$ or more, ${ }^{2-5}$ whereas the frequency reported in American studies is lower ${ }^{6-10}$ possibly reflecting geographical or ethnic differences in susceptibility ${ }^{111}$ and the application of varying diagnostic criteria. ${ }^{12} 13$

Multiple sclerosis is most likely to develop within the first 5 years $^{5-710}$ after optic neuritis, but the proportion of patients who develop disseminated lesions continues to rise with length of follow-up. ${ }^{14}$ Using an actuarial analysis it has been calculated that $60 \%$ of patients will have developed multiple sclerosis within 8 years, ${ }^{15}$ and $78 \%$ by 15 years, ${ }^{12}$ of the initial attack.

Several factors have provisionally been identified 
which may influence outcome in patients with optic neuritis. They include age of onset and sex, ${ }^{9}$ presence of oligoclonal immunoglobulins in cerebrospinal fluid $^{16}$ and recurrence. ${ }^{91517}$ The risk of multiple sclerosis is lower after simultaneous than sequential bilateral optic neuritis in adults, but higher in both instances than following bilateral optic neuritis in childhood. ${ }^{12} 17$

The association between the HLA system and multiple sclerosis prompted immunogenetic studies in patients with optic neuritis. Arnason et $a^{18}$ found no significant associations with antigens of the HLA-A and -B series, whereas Platz et al ${ }^{19}$ reported that the frequencies of HLA-A3, B7 and Ld-7a (Dw2) were similar in patients with multiple sclerosis and optic neuritis. Subsequent studies in patients with optic neuritis have demonstrated frequencies of these antigens which are intermediate between patients with multiple sclerosis and controls; ${ }^{20}$ the risk of multiple sclerosis developing after optic neuritis is increased four-fold in individuals positive for the B-cell antigen, BT 101 (broadly equivalent to DR2) and seven and a half times if clinical and laboratory risk factors are combined. ${ }^{15}$ More recently, Fielder et $a l^{21}$ found a significantly reduced frequency of the $\mathrm{BfF}$ allele in patients with clinically definite multiple sclerosis compared with optic neuritis and suggested that the presence of this Class III gene product might in some way protect against dissemination of demyelination outside the optic nerves.

In the present investigation we have reviewed all patients available from our earlier study, ${ }^{15}$ in order to determine the further incidence of multiple sclerosis over a longer period of observation and to reassess previously identified risk factors following changes in definition of the HLA-D region and availability of more discriminating serological reagents. ${ }^{22}$

\section{Patients and methods}

One hundred and forty six patients who had attended the physicians clinics at Moorfields Eye Hospital, City Road and High Holborn, with isolated optic neuritis, during the preceding 23 years, were reassessed clinically and tissuetyped, between August 1976 and August 1977, to document the subsequent occurrence of neurological symptoms in the optic nerves or elsewhere. The diagnosis of optic neuritis in the initial study conformed to the criteria of Earl and Martin $^{23}$ and non-caucasoid patients were excluded as were those with evidence of a condition other than multiple sclerosis which might cause an optic nerve lesion, onset of symptoms outside the age limits of 10-50 years, or development of symptoms while the patient was outside Europe.

One hundred and one of $146(69 \%)$ patients were reviewed between January 1983 and January 1985; of the 45 remaining individuals, 21 refused re-examination, three patients had died and 21 could not be traced. At reassessment patients were classified as having developed clinically definite or probable multiple sclerosis (ON/MS), using the same criteria as in $1977,{ }^{24}$ or as still having optic neuritis only (ON-only). Recurrent or bilateral optic neuritis was diagnosed only when there was a clear history of new visual symptoms not attributable to the initial lesion and these patients were classified as having optic neuritis only. In patients who developed multiple sclerosis only recurrences of optic neuritis which occurred before other signs of demyelination developed were included.

One hundred and sixty four normal Caucasian volunteers, mostly laboratory workers from south-east of England, were tissue-typed for comparison with the clinical groups.

$H L A$ typing All 101 patients and 164 controls were tissuetyped for Class I antigens (HLA-A, -B and -Cw) using a standard microlymphocytotoxicity test. ${ }^{25}$ HLA-DR and DQ typing was performed, by the same method, on enriched B-lymphocyte preparations. ${ }^{26}$ Sixteen HLA-A, 32 HLA-B, 7 HLA-Cw, 10 HLA-DR and 3 HLA-DQw specificities were defined using antisera obtained locally or donated by other typing laboratories. All had been evaluated against Histocompatibility Workshop reagents. In most instances at least four well-defined antisera were used for the detection of each allele and positive typing for any antigen was based on at least two concordant reactions. At the beginning of this study there were insufficient antisera to define DQw2 and DQw3, therefore data for these specificities are omitted from the final analysis. Only 120 of the control subjects were typed for DQw1.

All patients and 78 normal individuals from the control population were also typed for Factor B (Bf) alleles by high voltage agarose electrophoresis of serum samples followed by immunofixation. ${ }^{27}$

Statistics The significance of differences in frequency of antigens found in the groups was tested by the Chi-square test with Yates correction or by Fisher's exact test ${ }^{28}$ if the frequency of an antigen was small in any group (that is, less than $5 \%$ ). In view of the known association between multiple sclerosis and HLA-DR2 no correction was made for the number of antigens tested.

The relative risk (RR) for developing multiple sclerosis was calculated according to Svejgaard et al. ${ }^{29}$ Actuarial analysis ${ }^{30}$ was used to correct for varying length of followup between patients. The significance of the differences between actuarial curves was analysed by the method of Peto et al. ${ }^{31}$

\section{Results}

\section{Clinical assessment}

A flow diagram is shown (fig 1) indicating the number of patients seen in the initial review (1976-77), how they were then classified, and the results of review during 1983-85. Eighty eight out of the 146 patients were originally classified as having optic neuritis only (ON-only) of whom we were able to reassess $67(76 \%)$ after an interval of 5.8-30.5 years (median $10 \cdot 1$ years, mean 11.3 years) from the original episode. Twenty four of the 67 patients $(36 \%)$ had experienced neurological symptoms or developed neurological signs outside the optic nerves since our previous 


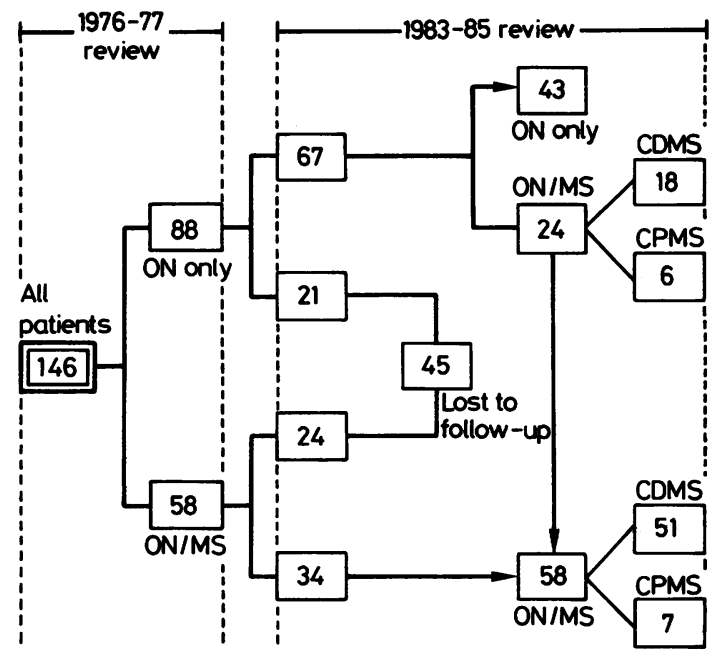

Fig 1 Flow diagram showing the number of patients and their classification after review in successive surveys.

assessment and could now be classified as having clinically definite (CDMS; 18/24) or clinically probable multiple sclerosis (CPMS; 6/24). The interval between optic neuritis and appearance of additional neurological features leading to the diagnosis of $C D$ or CPMS ranged from 3 months to 14 years (mean 5.6 years). The mean age of the two groups was very similar $(\mathrm{ON} / \mathrm{MS}=32.5$ years; $\mathrm{ON}$-only $=35$ years $)$.

Fifty eight out of 146 patients were originally classified as having developed multiple sclerosis; $34 / 58(59 \%)$ were reviewed and the diagnosis confirmed. Nine, of 10 patients, classified as clinically probable multiple sclerosis in the original series had developed further neurological features and could now be classified as having clinically definite multiple sclerosis. The remaining patient had had no further neurological episodes since the last review.

The 45 patients lost to follow-up consisted of $21 / 88$ $(24 \%)$ patients from the original ON-only group and $24 / 58(41 \%)$ patients with ON/MS. This bias in patient recall $(p<0.05)$ should be considered when interpreting our present results.

Overall, 58/101 (57\%) patients had developed multiple sclerosis by $1983-85$ after a mean follow-up period of 11.6 years (range 5.9-30.6 years), and 51 $(88 \%)$ of these patients had clinically definite disease. This compared with $58 / 146(40 \%)$ patients, of whom $62 \%$ had clinically definite disease, after 3.9 years (range 0.9-23 years), in the first survey. 24/45 (53\%) patients not reviewed in 1983-85 were already known to have developed multiple sclerosis at the time of the first survey. The minimum observed proportion of patients developing multiple sclerosis after nearly 12 years of follow-up (57\%) is similar to the figure pre- dicted at 12 years by actuarial analysis $(61 \%$, fig 2$)$ which also suggests that another $14 \%$ of patients will develop multiple sclerosis over the next 3 years, making a total of $75 \% 15$ years after the initial episode of optic neuritis.

\section{HLA data}

There was an increased frequency of HLA-DR2 $(p<$ $0.01 ; R R=2.9)$ and the phenotype HLA-B7-DR2 (p $<0.05$; $R R=2.5$ ) in patients with ON-only compared with healthy controls (table 1). In patients who had developed multiple sclerosis there was an increased frequency of HLA-B7 ( $<<0.01 ; R R=$ 2.5 ) and a highly significant increase in HLA-DR2 (p $<0.001 ; R R=4.9)$ compared with controls. The frequency of DR2 $(\mathbf{4 4 \% )}$ was lower in the group with ON-only than in patients with ON/MS (57\%), but the difference was not significant. No difference was observed when actuarial curves for developing multiple sclerosis were plotted for DR2 + ve and - ve patients (DR2 - ve curve not shown in fig 2).

HLA-DR3 alone, or in combination with B8, was also significantly increased in the ON/MS group compared with ON-only patients or normal controls (table 1). The frequency of DR2 and DR3 in

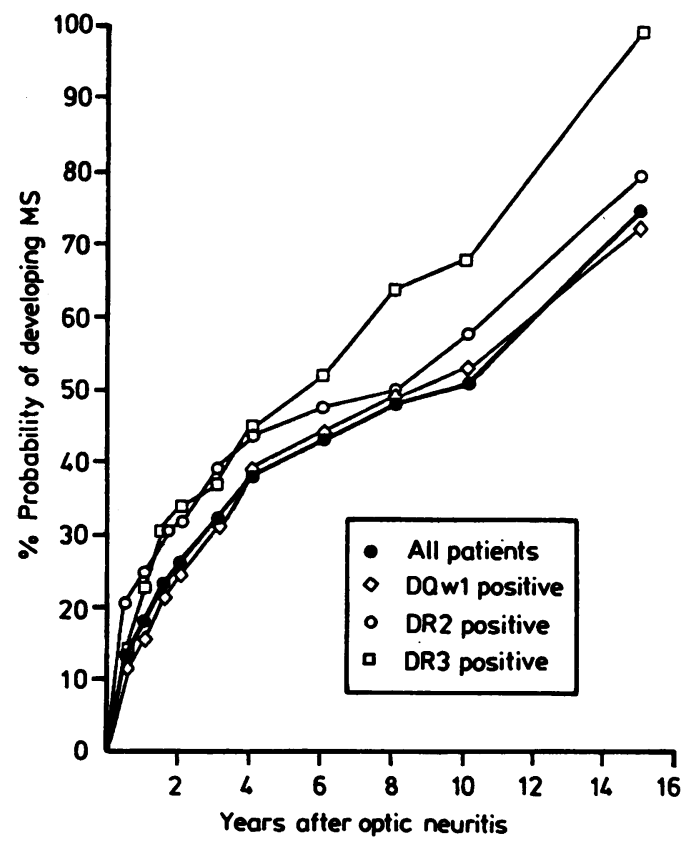

Fig 2 The percentage probability of developing multiple sclerosis for up to 16 years after an attack of optic neuritis in all patients and patients positive for $D R 2, D R 3$ and $D Q w 1$ respectively. 
Table 1 HLA antigen frequencies in patients with isolated optic neuritis, multiple sclerosis following optic neuritis and normal control subjects

\begin{tabular}{|c|c|c|c|c|c|c|}
\hline & \multirow{2}{*}{$\begin{array}{l}\text { ON-only }(A) \\
n=43 \\
(\%)\end{array}$} & \multirow{2}{*}{$\begin{array}{l}O N / M S(B) \\
\substack{n=58 \\
(\%)}\end{array}$} & \multirow{2}{*}{$\begin{array}{l}\text { Controls }(C) \\
n=164 \\
(\%)\end{array}$} & \multicolumn{3}{|l|}{$x^{2}$} \\
\hline & & & & $A \vee B$ & $A v C$ & $B v C$ \\
\hline $\begin{array}{c}\text { HLA-A1 } \\
\text { A3 } \\
\text { B7 } \\
\text { B8 } \\
\text { DR2 } \\
\text { DR3 } \\
\text { DQw1 } \\
\text { B7-DR2 } \\
\text { A3-B7-DR2 } \\
\text { B8-DR3 } \\
\text { A1-B8-DR3 } \\
\text { DR2-DR3 }\end{array}$ & $\begin{array}{r}35 \\
30 \\
40 \\
16 \\
44 \\
16 \\
56 \\
28 \\
9 \\
12 \\
12 \\
2\end{array}$ & $\begin{array}{l}41 \\
38 \\
45 \\
35 \\
57 \\
36 \\
64 \\
38 \\
22 \\
28 \\
24 \\
14\end{array}$ & $\begin{array}{l}29 \\
29 \\
24 \\
18 \\
21 \\
20 \\
47 \ddagger \\
13 \\
6 \\
13 \\
12 \\
0.5\end{array}$ & $\begin{array}{l}- \\
\overline{-} \\
5 \cdot 2^{*} \\
\frac{5 \cdot 9^{*}}{-} \\
\overline{-} \\
\overline{-} \\
t^{*}\end{array}$ & $\begin{array}{l}\bar{Z} \\
\bar{E} \\
8 \cdot 1^{* *} \\
\overline{-} \\
4 \cdot 2^{*} \\
\overline{-} \\
=\end{array}$ & $\begin{array}{l}- \\
\overline{7} \cdot 6^{* *} \\
6 \cdot 1^{*} \\
23 \cdot 8^{* * *} \\
5 \cdot 2^{*} \\
3 \cdot 9^{*} \\
14 \cdot 7^{* * *} \\
10 \cdot 6^{* *} \\
5 \cdot 7^{*} \\
4 \cdot 4^{*} \\
16 \cdot 0^{* * *}\end{array}$ \\
\hline
\end{tabular}

${ }^{*} \mathrm{p}<0.05 ; *{ }^{*} \mathrm{p}<0.01 ; * * \mathrm{p}<0.005$.

†Fisher Exact Probability Test.

$\pm n=120$.

$-=$ not significant

combination was significantly raised in patients with ON/MS compared with the ON-only group (p < $0.05 ; R R=6.7)$ or normal controls ( $p<0.001$; RR $=26$, see table 1). Although the presence of DR3 appeared overall to be associated with an increased risk of developing multiple sclerosis, the difference between the DR3 + ve and DR3 - ve actuarial curves (DR3 - ve curve not shown in fig 2) was not statistically significant $\left(\chi^{2}=3.04 ; 0.10>p>0.05\right)$ perhaps reflecting the small number of cases we have been able to study beyond 10 years follow-up. When the actuarial curves were extended to 15 years, it was predicted that $99 \%$ of DR3 + ve and $63 \%$ of DR3 - ve individuals would have developed multiple sclerosis.

Although there was an increased frequency of HLA-DQw1 in patients with multiple sclerosis compared with controls ( $p<0.05, R R=1.5$ ), actuarial analysis showed no difference in the risk of developing multiple sclerosis between DQw1 + ve and - ve individuals (DQw1 - ve curve not shown). The relationship between BT 101, DQw1 and DR2 is shown in table 2, based on typing results in the same individuals from 1978 and the present review, BT 101 occurred most frequently followed by DQwl and then DR2. The combined frequency of DQw1 and DR2 was equal to the frequency of BT 101. The frequency of BT 101 in the original $58 \mathrm{ON} / \mathrm{MS}$ patients and 88 with $\mathrm{ON}$-only was $84 \%$ and $54 \%$ respectively; corresponding frequencies in the sample of the cases we have since reassessed is $74 \%$ and $63 \%$ respectively.

The frequency of the BfF phenotype (including both the BfFF homozygotes and the BfSF and BfFF1 heterozygotes) showed no significant variation between patients with ON/MS and ON-only (table 3).

\section{Recurrent attacks of optic neuritis}

Thirty six of $101(36 \%)$ patients had more than one episode of optic neuritis and in 23/36 the recurrence affected the other eye. Recurrent attacks occurred in $16 / 43(37 \%)$ patients in the $\mathrm{ON}$-only group compared with $20 / 58(34 \%)$ patients who subsequently developed multiple sclerosis ( $p=$ NS; table 4). In 1978, $17 / 88(19 \%)$ patients with ON-only and $36 / 58(62 \%)$ with $\mathrm{ON} / \mathrm{MS}$ had recurrent optic neuritis and thus at that time recurrence appeared to be a useful risk factor for the development of multiple sclerosis.

It was also shown, in the earlier study, that patients who experienced a recurrent attack of optic neuritis and were BT 101 positive were more likely to develop multiple sclerosis than those patients who were BT 101 negative. In the present series, the presence of neither DR2 nor DQw1 had prognostic significance for the development of multiple sclerosis (see table 4). The increased risk of developing multiple sclerosis in individuals carrying HLA-DR3 was not further influenced by recurrent episodes of optic neuritis (table 4).

Table 2 Frequency of BT 101 in $O N$-only and ON/MS patients compared with the frequencies of DQw1 and DR2

\begin{tabular}{llllll}
\hline Groups & $B T 101^{*}$ & $D Q w 1$ & $D R 2$ & $D Q w 1 \pm D R 2$ & Total \\
\hline ON-only & $27(63 \%)$ & $24(56 \%)$ & $19(44 \%)$ & $27(63 \%)$ & 43 \\
ON/MS & $43(74 \%)$ & $37(64 \%)$ & $33(57 \%)$ & $43(74 \%)$ & 58 \\
\hline
\end{tabular}

*Data from 1978 study. 
Table 3 Frequencies of $B f$ phenotypes in patients with isolated optic neuritis, multiple sclerosis following optic neuritis and normal control subjects

\begin{tabular}{|c|c|c|c|c|c|c|}
\hline & \multirow{2}{*}{$\begin{array}{l}\text { ON-only }(A) \\
n=43 \\
(\%)\end{array}$} & \multirow{2}{*}{$\begin{array}{l}O N / M S(B) \\
n=58 \\
(\%)\end{array}$} & \multirow{2}{*}{$\begin{array}{l}\text { Controls }(C) \\
n=78 \\
(\%)\end{array}$} & \multicolumn{3}{|l|}{$\chi^{2}$} \\
\hline & & & & $A \cup B$ & $A \vee C$ & $B \vee C$ \\
\hline Bf-SS & 58 & 65 & 64 & - & - & - \\
\hline $\begin{array}{l}\text { SF } \\
\text { FF/FF1 }\end{array}$ & 26 & 26 & 29 & - & - & - \\
\hline $\begin{array}{l}\text { FF/FFI } \\
\text { SIF }\end{array}$ & 14 & - & $\underline{6}$ & 二 & Z & 二 \\
\hline SF1 & 2 & 5 & - & - & - & - \\
\hline $\begin{array}{l}\text { S1F1 } \\
\text { S1S1 }\end{array}$ & $=$ & $\overline{2}$ & $=$ & $=$ & $\bar{z}$ & $=$ \\
\hline \multirow[t]{2}{*}{ All F phenotypes* } & & & & & & \\
\hline & 42 & 37 & 36 & - & - & - \\
\hline
\end{tabular}

*Includes SF and FF1 heterozygotes and FF homozygotes.

$-=$ not significant.

\section{Discussion}

In 1978 we observed that $40 \%$ of 146 patients with optic neuritis had developed multiple sclerosis after a mean interval of 3.9 years and identified three risk factors for this event. Since our original survey a further third of the patients whom we have been able to review have developed multiple sclerosis and the overall minimum observed frequency of multiple sclerosis is now $57 \%$ after 11.6 years follow-up; this is similar to the figure previously predicted $(60 \%)$ at 8 years using actuarial methods. Applying this type of analysis to the present group it is predicted that a further $18 \%$ of patients with optic neuritis $(75 \%$ overall) will develop multiple sclerosis after 15 years follow-up.

Table 4 Number of cases with unilateral or recurrent $O N$ in $O N$-only and $O N / M S$ patients each divided into DR2, DR3 and $D Q w 1$ positive groups

\begin{tabular}{|c|c|c|c|}
\hline & Unilateral & Recurrent & Total \\
\hline $\begin{array}{l}\text { All patients } \\
\text { ON-only } \\
\text { ON-MS }\end{array}$ & $\begin{array}{l}65(64 \%) \\
27(63 \%) \\
38(66 \%)\end{array}$ & $\begin{array}{l}36(36 \%) \\
16(37 \%) \\
20(34 \%)\end{array}$ & $\begin{array}{r}101 \\
43 \\
58\end{array}$ \\
\hline \multicolumn{4}{|c|}{$\chi^{2}=0.005 p=N S$} \\
\hline $\begin{array}{l}\text { DR2-positive } \\
\text { ON-only }\end{array}$ & $10(53 \%)$ & $9(47 \%)$ & 19 \\
\hline $\begin{array}{l}\text { DR2-positive } \\
\text { ON-MS }\end{array}$ & $19(59 \%)$ & $13(41 \%)$ & 32 \\
\hline Total & $29(57 \%)$ & $22(43 \%)$ & 51 \\
\hline \multicolumn{4}{|c|}{$\chi^{2}=0.03 p=N S$} \\
\hline $\begin{array}{l}\text { DQw1-positive } \\
\text { ON-only }\end{array}$ & $15(62 \%)$ & $9(38 \%)$ & 24 \\
\hline $\begin{array}{l}\text { DQwl-positive } \\
\text { ON-MS }\end{array}$ & $27(73 \%)$ & $10(27 \%)$ & 37 \\
\hline Total & $42(69 \%)$ & $19(31 \%)$ & 61 \\
\hline \multicolumn{4}{|c|}{$\chi^{2}=0.34 \mathrm{p}=\mathrm{NS}$} \\
\hline $\begin{array}{l}\text { DR3-positive } \\
\text { ON only }\end{array}$ & $4(57 \%)$ & $3(43 \%)$ & 7 \\
\hline $\begin{array}{l}\text { DR3-positive } \\
\text { ON-MS }\end{array}$ & $14(66 \%)$ & $7(34 \%)$ & 21 \\
\hline Total & $18(64 \%)$ & $10(36 \%)$ & 28 \\
\hline$\chi^{2}=0.2$ & NS & & \\
\hline
\end{tabular}

This figure approximates closely to that predicted by Hutchinson ${ }^{12}$ for Northern Ireland, using a similar analysis (78\% at 15 years). But our figures for observed and predicted conversion to multiple sclerosis are falsely low because a significantly higher proportion of $\mathbf{4 5}$ patients lost to follow-up were from the group who had already developed multiple sclerosis at the time of the first survey. If these patients are taken into consideration the observed and predicted frequencies of conversion to multiple sclerosis are both higher and approach the observed figure of $85 \%$ in McAlpine's ${ }^{2}$ reassessment of Lynn's ${ }^{32}$ data.

We found significantly raised frequencies of HLA- $\triangle$ DR2 in patients with isolated optic neuritis $(p<0.01$; $R R=2.9$ ) and in those who subsequently developed multiple sclerosis $(p<0.005 ; R R=4.9)$ comparedo with the normal population. The higher frequencies and relative risks in the patients who developed multiple sclerosis suggest that HLA-DR2 may be a significant risk factor for the development of dissemination of lesions, but the difference was not significant. While DR2 was associated with an increased susceptibility to both optic neuritis and multiple sclerosis, on the present evidence, it did not appear to influence long term progression from the former to the latter.

HLA-DR3 was increased in the group of patients with ON/MS but not in patients with ON-only. This association could not be examined in 1978. Based on actuarial analysis over $95 \%$ of DR3 + ve patients will have developed multiple sclerosis within 15 years compared with $63 \%$ of those without this antigen. Patients who possessed both DR2 and DR3 were even more likely to develop multiple sclerosis. This finding supports the suggestion of Madigand et al $^{33}$ that independent genes linked to DR2 and DR3 complement each other in determining susceptibility to multiple sclerosis. If confirmed in a larger study, the question would arise whether this subgroup of patients, with such a high relative risk for developing multiple sclerosis after optic neuritis, might, when an 
effective form of treatment is developed, benefit from its institution during the presenting episode.

It is of interest that HLA-DQwl shows a significantly increased frequency in patients with ON/MS. We found an even higher association between this antigen and all forms of multiple sclerosis in the Grampian region of North East Scotland. ${ }^{34}$ The frequency of HLA-DR2 in patients with multiple sclerosis in the Grampian region was high (49\%), but similar to that found in a local control population (41\%).

In 1978, progression of isolated optic neuritis to multiple sclerosis appeared to correlate with the presence of the B lymphocyte alloantigen, BT 101. At the 1978 International Histocompatibility Workshop the sera defining BT 101 were found to correlate significantly with DR2, but further reactions were also found with cells from HLA-DR1 and DRw6 positive individuals. HLA-DR $1,-2$, and -w6 are now known to be in linkage disequilibrium with the recently defined antigen $\mathrm{DQw1},{ }^{21}$ and the sera used to define BT 101 ("BEW" and "SEW") have been found to correlate more closely with this antigen than with DR2 (unpublished data). This is consistent with our findings which suggest that the sera defining BT 101 are of broad specificity reacting with both DR2 and DQw1 positive cells but correlating more closely with DQw1.

BT 101 was associated with an increased risk of developing multiple sclerosis in the earlier study but this finding is not confirmed in the present study in which the follow-up has been extended. There are several possible reasons for these conflicting results. First, since 1978, there have been advances in understanding the structure of the HLA system; new methods and reagents are now available for defining Class II antigens. Seven patients classified as BT 101 positive in 1978 are negative for both HLA-DQwl and HLA-DR2. Conversely, six patients classified as BT 101 negative in 1978 are positive for HLA-DQw1, and/or HLA-DR2, using modern typing reagents. Studies carried out at different times and using different reagents cannot be easily compared. A second important factor is the length of follow-up which has been extended from a mean of 4 years, in the original study, to 11.6 years in the present study. The separation of the actuarial curves plotted for cases with and without BT 101 during the initial follow-up have not been maintained beyond 10 years so that overall the difference is no longer observed; given the high rates of conversion to multiple sclerosis after 10 years the later parts of the curve are necessarily based on fewer observations, especially in patients with $\mathrm{ON}$ only, and are therefore subject to the effect of sampling errors; in our recent review $40 \%$ of the original $\mathrm{ON} / \mathrm{MS}$ group but only $23 \%$ of the $\mathrm{ON}$-only cases could not be reassessed.

The incidence of "observed" cases of multiple sclerosis developing after optic neuritis in the former study $(\mathbf{4 0 \%})$, was less than that observed in other studies from the United Kingdom with a longer period of follow-up. ${ }^{45}$ For example, McAlpine ${ }^{2}$ reanalysed Lynn's ${ }^{32}$ original data (which showed a $50 \%$ overall incidence of multiple sclerosis), by excluding all cases of optic neuritis followed-up for less than 5 years, and found that a much higher percentage of patients $(85 \%)$ had developed multiple sclerosis. In our study, since $75 \%$ of patients with optic neuritis may subsequently develop multiple sclerosis, even if all BT 101 positive cases convert, some BT 101 negative individuals must also develop multiple sclerosis as has in fact been observed.

What then is the significance of the documented association with DR2? It is clear from our study that DR2 is associated with demyelinating lesions whether they are confined to the optic nerve or are more widespread in the CNS but on the present evidence its presence does not appear to influence long term conversion of the isolated to disseminated form of disease. Additional factors might be important and we have produced evidence implicating HLA-DR3, whether it is present in isolation or in combination with HLA-DR2. Factor B alleles, previously thought to influence progression to multiple sclerosis, ${ }^{21}$ appear not to be important in this respect. Our present results are supported by findings in a larger group of patients, from the Grampian region of Scotland, in which no significant deviation of the distribution of $\mathrm{Bf}$ alleles was found between groups of patients with markedly contrasting clinical courses.

Thirty six per cent of all patients reviewed in the present series had recurrent optic neuritis, and of these, just over one half $(55 \%)$ went on to develop multiple sclerosis. These figures are similar to those in the original study (31\% and $63 \%$ respectively). In 1978 few patients in the ON-only group experienced recurrent attacks (19\%). As a result, at this early stage of follow-up, recurrent attacks appeared to be a significant risk factor. Longer follow-up indicates that more patients with isolated optic neuritis will experience a second attack ( $37 \%$ in the present study) if followed for a sufficient length of time. Whether they will later develop lesions outside the optic nerves remains to be determined.

Several conclusions can be drawn from our two surveys on the relationship between optic neuritis, multiple sclerosis and the HLA system. The proportion of patients who develop multiple sclerosis after an attack of optic neuritis increases with length of follow-up; the risk does not decrease significantly with time and the chance that a patient with optic neuritis will escape multiple sclerosis over the suc- 
ceeding 15 years is less than 1 in 4 . This fact alone makes investigation of long-term risk factors difficult and we have not been able to demonstrate, with confidence, clinical or laboratory markers of increased risk applicable to the individual patient (the effect of HLA-DR3 awaits confirmation).

One of the strengths of our two studies is the length of follow-up but herein also lies its weakness. An increasing number of patients has defaulted with time and there have been important technical advances in HLA typing: it would clearly have been inappropriate to revert to less precise methods for the second study. It is therefore of particular significance that despite these difficulties some findings are constant - the increasing frequency of multiple sclerosis with time (the percentage predicted in 1978 is now observed) and the association of both optic neuritis and multiple sclerosis with HLA-DR2. The failure to confirm that recurrence of optic neuritis or DR2 positivity increases the risk of subsequent development of multiple sclerosis is perhaps not surprising for several reasons. First, our original 146 patients were not recruited prospectively from a population base but were selected retrospectively from individuals already attending hospital clinics. It is likely that selective biases were operating even at that stage and these are known to have been compounded in tracing patients for the most recent review. Secondly, the study of optic neuritis and multiple sclerosis by MRI has shown that the clinical method for determining the sequential development of multiple lesions outside the optic nerve (necessary for the diagnosis of multiple sclerosis in the present context) is much less reliable than had been supposed; $70 \%$ of patients with isolated optic neuritis defined clinically, as in this study, have MRI lesions outside the visual system at presentation (D H Miller; personal communication) and in a sequential study of acute isolated optic neuritis it was found that $20 \%$ (5/25 patients) developed new MRI lesions over a mean of 8 months, none of whom had experienced new symptoms. ${ }^{35}$ The systematic application of this approach combined with HLA typing in a prospective study would be the best way currently available for assessing the significance of risk factors in isolated optic neuritis.

This work was supported by a grant from the Medical Research Council. We thank Miss E Bolton, Mrs S Smithers and Mrs S Hing for their excellent technical assistance and Mrs N Fisher for typing the manuscript.

\section{References}

1 McDonald WI. Doyne Lecture: The significance of optic neuritis. Trans Ophthalmol Soc UK 1983;103:230-46.

2 McAlpine D. The benign form of multiple sclerosis: re- sults of a long-term study. $\mathrm{Br}$ Med $J$ 1964;2:1029-32.

3 Nikoskelainen E, Reikkinen P. Optic neuritis-a sign of multiple sclerosis or other diseases of the central nervous system. A retrospective analysis of 116 patients. Acta Neurol Scand 1974;50:690-718.

4 Perkin GD, Rose FC, eds. Optic Neuritis and its Differential Diagnosis. Oxford: Oxford University Press, 1979:292.

5 Bradley WR, Whitty CWM. Acute optic neuritis: prognosis for development of multiple sclerosis. $J$ Neurol Neurosurg Psychiatry 1968;31:10-8.

6 Kurland LT, Beebe GW, Kurtzke JF, et al. Studies on the natural history of multiple sclerosis. 2. The progression of optic neuritis to multiple sclerosis. Acta Neurol Scand 1966;42, Supplement 19:157-76.

7 Percy AK, Norbrega FT, Kurland LT. Optic neuritis and multiple sclerosis. Arch Ophthalmol 1972;87: 135-9.

8 Taub RG, Rucker TW. The relationship of retrobulbar neuritis to multiple sclerosis. Am J Ophthalmol 1954;37:494-7.

9 Cohen MM, Lessell S, Wolf PA. A prospective study of the risk of developing multiple sclerosis in uncomplicated optic neuritis. Neurology 1979;29:208-13.

10 Collis WJ. Acute unilateral retrobulbar neuritis. Arch Neurol 1965;13:409-12.

11 Shibasaki H, McDonald WI, Kuroiwa Y. Racial modification of clinical picture of multiple sclerosis: comparison between British and Japanese patients. $J$ Neurol Sci 1981;49:253-71.

12 Hutchinson WM. Acute optic neuritis and the prognosis for multiple sclerosis. J Neurol Neurosurg Psychiatry 1976;39:283-9.

13 Ebers GC. Optic neuritis and multiple sclerosis. Arch Neurol 1985;42:702-4.

14 McAlpine D, Lumsden CE, Acheson ED. eds. Multiple Sclerosis. A Re-appraisal. Edinburgh: Livingstone, 1965.

15 Compston DAS, Batchelor JR, Earl CJ, McDonald WI. Factors affecting the risk of multiple sclerosis developing in patients with optic neuritis. Brain 1978; 101:495-511.

16 Moulin D, Paty DW, Ebers GC. The predictive value of cerebrospinal fluid electrophoresis in 'possible' multiple sclerosis. Brain 1983;106:809-16.

17 Parkin PJ, Hierons R, McDonald WI. Bilateral optic neuritis: a long-term follow up. Brain 1984;107: 951-64.

18 Arnason BGW, Fuller TC, Lehrich JR, Wray SH. Histocompatibility types and measles antibodies in multiple sclerosis and optic neuritis. J Neurol Sci 1974; 22:419-28.

19 Platz P, Ryder LP, Staub-Nielsen L, Svejgaard A, Thomsen M, Sandberg-Woolheim M. HL-A and idiopathic optic neuritis. Lancet 1975;i:520-1.

20 Stendahl-Brodin L, Link H, Moller E, Norrby E. Optic neuritis and distribution of genetic markers of the HLA system. Acta Neurol Scand 1978;53:418-31.

21 Fielder AHL, Batchelor JR, Vakarelis BN, Compston DAS, McDonald WI. Optic neuritis and multiple sclerosis: do Factor $B$ alleles influence progression of disease. Lancet 1981;ii:1246-8. 
22 Albert ED, Baur MP, Mayr WR, eds. Nomenclature for factors of the HLA system 1984. In: Histocompatibility Testing 1984. Berlin, Heidelberg, New York and Tokyo: Springer-Verlag, 1985:4-8.

23 Earl CJ, Martin B. Prognosis in optic neuritis related to age. Lancet 1967;i:74-6.

24 McDonald WI, Halliday AM. Diagnosis and classification of multiple sclerosis. Br Med Bull 1977;33:4-8.

25 Bodmer JG, Pickbourne P, Richards S. Ia serology. In: Bodmer WF, Batchelor JR, Bodmer JG, Festenstein H, Morris PJ, eds. Histocompatibility Testing 1977. Copenhagen: Munksgaard, 1978:35-84.

26 Mann DL, Abelson L, Henkart P, Harris S, Amos BD. Serological detection of B lymphocyte antigens. In: Kissmeyer-Nielson F, ed. Histocompatibility Testing (1975). Copenhagen: Munksgaard, 1975:705-7.

27 Alper CA, Boenisch T, Watson L. Genetic polymorphism in human glycine rich beta glycoprotein. $J$ Exp Med 1972;135:68-80.

28 Armitage P, ed. Statistical Methods in Medical Research. Oxford: Blackwell, 1971.

29 Svejgaard A, Hauge M, Jersild C, et al. The HLA system.
An introductory survey. In: Beckman L, Hauge M, eds. Monographs in Human Genetics. Basel: Karger, 1975:1-103.

30 Barnes BA. Survival date of renal transplant in patients. Transplantation 1965;3:812-21.

31 Peto R, Pike MC, Armitage P, et al. Design and analysis of randomised clinical trials requiring prolonged observation of each patient. Part II: Analysis and examples. $\mathrm{Br} J$ Cancer 1977;35:1-36.

32 Lynn BH. Retrobulbar neuritis. Trans Ophthalmol Soc UK 1959;79:701-16.

33 Madigand M, J-Foger J, Fauchet R, Sabouraud O, Genetet B. HLA profiles in multiple sclerosis suggest two forms of disease and the existence of protective haplotypes. J Neurol Sci 1982;53:519-29.

34 Francis DA, Batchelor JR, McDonald WI, Downie AW, Hern JEC. Multiple sclerosis and HLA-DQw1. Lancet 1986;i:211.

35 Miller DH, Ormerod IEC, McDonald WI, et al. A clinical and MRI follow-up study of isolated optic neuritis. In: Society of Magnetic Resonance in Medicine; Book of Abstracts 1986;3:774-5. 